УДК 538.11

DOI 10.18101/2306-2363-2020-2-3-6

\title{
РАСЧЕТЫ СИЛОВЫХ И УПРУГИХ ПОСТОЯННЫХ $\alpha$-Fе ИЗ ЭКСПЕРИМЕНТАЛЬНОГО ФОНОННОГО СПЕКТРА
}

\section{(C) Бадмаараг А.}

научный сотрудник,

Объединенный институт ядерных исследований

141980, Дубна, Россия

badmaarag0815@gmail.com

\section{(C) Лыгденов В. Ц.}

научный сотрудник,

Бурятский государственный университет имени Доржи Банзарова

670000, Улан-Удэ, Россия

Институт физического материаловедения СО РАН

670031, Улан-Удэ, Россия

lygdenov65@mail.ru

\section{(С) Гантулга Ц.}

кандидат физико-математических наук,

Монгольский государственный университет

210646, Улан-Батор, Монголия

E-mail: gantulga@smcs.num.edu.mn

\section{(C) Энхтор Л.}

кандидат физико-математических наук,

Монгольский государственный университет

210646, Улан-Батор, Монголия

E-mail: enkhtor@num.edu.mn

Рассчитаны значения радиальных и тангенциальных силовых постоянных межатомного взаимодействия на первых шести координационных сферах $\alpha$-Fe из фононного спектра, сопоставлены с ранее полученными данными. Расчеты проводились методом наименьших квадратов путем описания экспериментальных фононных ветвей с помощью динамической матрицы, которая построена в модели Де Лане. Для проверки достоверности полученных значений силовых постоянных с их применением построены фононные спектры и рассчитаны упругие постоянные $\alpha-\mathrm{Fe}$, которые удовлетворительно согласуются с соответствующими экспериментальными данными.

Ключевые слова: радиальные силовые постоянные; тангенциальные силовые постоянные; динамическая матрица; фононный спектр; упругие постоянные; координационные сферы; $\alpha$-Fe.

\section{Для цитирования:}

Бадмаараг А., Лыгденов В. Ц., Гантулга Ц., Энхтор Л. Расчеты силовых и упругих постоянных $\alpha$-Fe из экспериментального фононного спектра // Вестник Бурятского государственного университета. Химия. Физика. 2020. Вып. 2. С. 3-6. 
В [1] методом неупругого рассеяния нейтронов был измерен фононный спектр $\alpha$-Fe в направлениях симметрии при температуре 280 К и описан с учетом силовых постоянных Борна-Кармана на первых пяти координационных сферах. В [1] не была оценена достоверность полученных силовых постоянных путем расчета с их применением значений упругих постоянных $\alpha-\mathrm{Fe}$.

Фононный спектр $\alpha-\mathrm{Fe}$, измеренный в [2] при 1173 К, был описан в [3] с учетом силовых постоянных Борна-Кармана на первых пяти координационных сферах. С применением последних рассчитаны значения упругих постоянных $\alpha-$ $\mathrm{Fe}$, которые заметно отличаются от экспериментальных значений, определенных в [4] методом ультразвуковых волн при температуре 1173 К.

Методика расчета радиальных и тангенциальных силовых постоянных металлов с объемно центрированной кубической структурой (ОЦК) из экспериметального фононного спектра описана в [5] с применением выражений для элементов динамической матрицы [6] в модели Де Лане. Выражения для расчетов упругих постоянных ОЦК металлов с применением значений радиальных и тангенциальных силовых постоянных межатомного взаимодействия даны в [6].

Таблица

Радиальные и тангенциальные силовые постоянные $\alpha-\mathrm{Fe}(\mathrm{H} / \mathrm{m})$

\begin{tabular}{|c|c|c|}
\hline $\begin{array}{c}\text { Номер } \\
\text { сферы } \mathrm{n}\end{array}$ & $\bar{\alpha}_{\mathrm{n}}$ & $\bar{\beta}_{\mathrm{n}}$ \\
\hline 1 & 45.51 & 2.51 \\
\hline 2 & 14.31 & 0.64 \\
\hline 3 & 1.27 & 0.52 \\
\hline 4 & -0.26 & 0.18 \\
\hline 5 & 0.26 & -0.81 \\
\hline 6 & 0.88 & -0.43 \\
\hline
\end{tabular}

В таблице даны значения радиальных $\bar{\alpha}_{\mathrm{n}}$ и тангенциальных $\bar{\beta}$ силовых постоянных $\alpha$-Fe на первых шести координационных сферах, полученныев данной работе методом наименьших квадратов из экспериментально измеренного в [1] фононного спектра при температуре 280 К (рис.). Для проверки достоверности полученных силовых постоянных $\alpha-\mathrm{Fe}$ мы рассчитали с их применением зависимости $v(q)$ дисперсии фононов, которые показаны на рисунке. Для сравнения там же квадратиками обозначены данные фононных частот, измеренные в [1]. Рассчитанные кривые фононных ветвей удовлетворительно совпадают с экспериментальными значениями фононных частот $\alpha-\mathrm{Fe}[1]$.

С применением полученных значений силовых постоянных, мы рассчитали значения упругих постоянных $\alpha-\mathrm{Fe}: C_{11}=24.5 \cdot 10^{10} \mathrm{H} / \mathrm{m} ; C_{12}=8.9 \cdot 10^{10} \mathrm{H} / \mathrm{m} ; C_{44}=$ $12.6 \cdot 10^{10} \mathrm{H} / \mathrm{M}$. Они удовлетворительно согласуются с экспериментальными значениями [7]: $C_{11}=23.3 \cdot 10^{10} \mathrm{H} / \mathrm{M} ; C_{12}=13.5 \cdot 10^{10} \mathrm{H} / \mathrm{M} ; C_{44}=11.8 \cdot 10^{10} \mathrm{H} / \mathrm{M}$. 


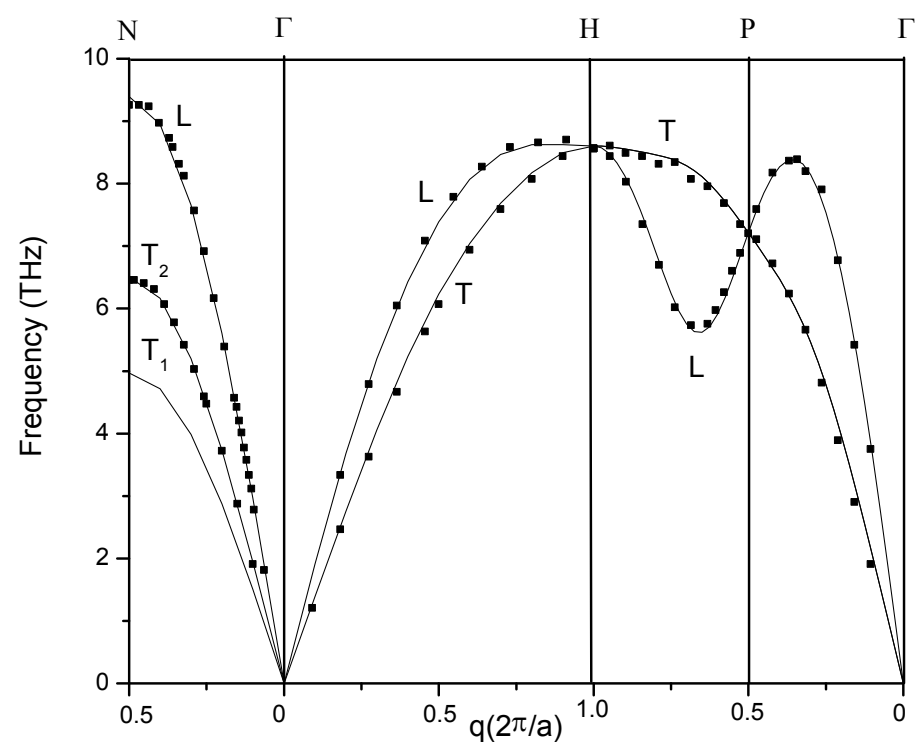

Рис. Фононный спектр $\alpha$-Fе при температуре 280 К. Экспериментальные значения частот [1] показаны квадратиками, а сплошные кривые соответствуют рассчитанным в данной работе фононным ветвям с учетом радиальных и тангенциальных силовых постоянных на первых шести координационных сферах.

\section{Литература}

1. Minkiewicz V. J., Shirane G., Natans R. Phonon dispersion for iron // Phys. Rev. 1967. - V. 162. - P. 528-537.

2. Neuhaus J., Petry W., Krimmel A. Phonon softening and martensitic transformation in $\alpha-F e / /$ Physica B. — 1997. — V. 234-236. — P. 897-913.

3. Leonov I., Poletaev A. I., Anosimov, D. Vollhardt V. I. Calculated phonon spectra of paramagnetic iron at the $\alpha-\gamma$ phase transition // Phys. Rev. B. - 2012. - V. 85. - P. 020401.

4. Dever D. J. Temperature dependence of the elastic constants in $\alpha$-iron single crystals: relationship to spin order and diffusion anomalies // J. Appl. Phys. — 1972. - V. 43. - P. 32933301.

5. Энхтор Л., Силонов В. М. Расчет радиальных и тангенциальных силовых постоянных цезия из экспериментального фононного спектра // Поверхность. Рентгеновские, синхротронные и нейтронные иссследования. — 2015. — № 12. - С. 5971.

6. Enkhtor L., Silonov V. M., Galbadrakh R. New general equation for calculations of elements of dynamical matrix and elastic constants of BCC and FCC metals // Int. Jour. Adv. Res. in Phys. Sci. - 2015. - V. 2(4). - P. 10-16.

7. Rayne J. A., Chandrasekhar B. S. Elastic Contants of Iron from 4.2 to $300 \mathrm{~K} / /$ Phys. Rev. - 1961. - V. 122. - P. 1714-1721. 


\section{CALCULATIONS OF FORCE AND ELASTIC CONSTANTS $\alpha$-Fe FROM EXPERIMENTAL PHONON SPECTRUM}

\section{Badmaarag A.}

researcher

Joint Insitute for Nuclear Research

141980, Dubna, Russia

badmaarag0815@gmail.com

Lygdenov $V . T_{S}$.

researcher

Banzarov Buryat State University

670000, Ulan-Ude, Smolina, str., 24a, Russia

Institute of Physical Materials Science SB RAS

70031, Ulan-Ude, Russia

lygdenov65@mail.ru

Gantulga Ts.

Candidate of Physical and Mathematical Sciences

National University of Mongolia

210646, Ulanbaatar, Mongolia

gantulga@smcs.num.edu.mn

\section{Enkhtor L.}

Candidate of Physical and Mathematical Sciences

National University of Mongolia

210646, Ulanbaatar, Mongolia

enkhtor@num.edu.mn

The values of the radial and tangential force constants of the interatomic interaction on the first six coordination spheres $\alpha$-Fe from the phonon spectrum are calculated and compared with the previously obtained data. The calculations were carried out by the method of least squares by describing the experimental phonon branches using a dynamic matrix, which is constructed in the De Lanet model. To verify the reliability of the obtained values of the force constants, phonon spectra are constructed and elastic constants $\alpha-\mathrm{Fe}$ are calculated, which are in satisfactory agreement with the corresponding experimental data.

Keywords: radial force constants; tangential force constants; dynamic matrix; phonon spectrum; elastic constants; coordination spheres; $\alpha-\mathrm{Fe}$.

Статья поступила в редакцию 16.03.2020; одобрена после рецензирования 3.09.2020; принята к публикации 30.10.2020. 